\title{
Red Meat Intake and Colorectal Cancer Risk: A Summary of Epidemiological Studies
}

\author{
Doris S. M. Chan • Dagfinn Aune • Teresa Norat
}

Published online: 16 December 2012

(C) Springer Science+Business Media New York 2012

\begin{abstract}
Epidemiological evidence of red meat and processed meat consumption and colorectal cancer risk has accumulated during the past decades. Meta-analyses of case-control and prospective cohort studies have shown a moderate increased risk, but the association is controversial. Because diet is one of the modifiable lifestyle factors for colorectal cancer prevention, the relationship has an important public health perspective. Three prospective cohort studies and one case-control study of total red meat and processed meat and colorectal cancer were published in 2011 and 2012. The findings were in general supportive of an increased risk with higher consumption. The same applies to the four studies each on fresh red meat and processed meat. Associations with dietary heterocyclic amines, nitroso-compounds, and heme iron intake are inconsistent, but evidence suggested a positive association between heme iron intake and colorectal cancer risk.
\end{abstract}

Keywords Epidemiological studies $\cdot$ Red meat $\cdot$ Processed meat $\cdot$ Colorectal cancer risk $\cdot$ Public health $\cdot$ Cancer prevention

\section{Introduction}

Colorectal cancer is the third most common cancer, with 1.2 million new cases diagnosed in 2008 worldwide [1]. Screening

\footnotetext{
D. S. M. Chan $(\bowtie) \cdot$ D. Aune $\cdot$ T. Norat

Department of Epidemiology and Biostatistics,

School of Public Health, Imperial College London,

St. Mary's Campus, Norfolk Place,

London, UK W2 1PG

e-mail: d.chan@imperial.ac.uk

D. Aune

e-mail: d.aune@imperial.ac.uk

T. Norat

e-mail: t.norat@imperial.ac.uk
}

and surveillance of adenomatous polyps has been put forward as the primary prevention. Lifestyle is thought to influence colorectal cancer risk; with the addition of lifestyle modification, colorectal cancer incident could be further reduced [2]. Hence, examining the diet-colorectal cancer associations has significant public health perspective.

Red meat and processed meat consumption and their role in colorectal cancer etiology have been widely studied in the past decades. The large body of evidence in humans encompasses mostly observational studies, with case-control, or prospective cohort designs, in different study populations. The definition for red meat varies between studies. Generally, it is defined as all fresh, minced, and frozen beef, veal, pork, and lamb, and processed meat preserved by the addition of preservatives or by marinating, smoking, salting, airdrying, heating, or methods other than freezing, which include ham, bacon, sausages, pate, and tinned meat.

At the ecological level, there is a positive association between red meat consumption and colorectal cancer incidence rates [3, 4] and mortality [5]. Summarizing findings from a number of case-control studies and/or prospective cohort studies, several meta-analyses have shown a positive association between red meat intake and colorectal cancer risk [6-8]. In 2010, we observed in our meta-analysis of 11 prospective studies, a moderate but statistically significant $14 \%$ increased risk $\left(95 \% \mathrm{CI}=4-24 \%, \mathrm{I}^{2}=56 \%\right)$ of colorectal cancer for every $100 \mathrm{~g} /$ day increase of red meat and processed meat consumption [9]. By meat type, the relative risk (RR) increase was $17 \%(95 \% \mathrm{CI}=5-31 \%$, $\mathrm{I}^{2}=0 \%, 8$ studies) for every $100 \mathrm{~g} /$ day fresh red meat and $18 \%\left(95 \% \mathrm{CI}=10-28 \%, \mathrm{I}^{2}=12 \%, 9\right.$ studies $)$ for every $50 \mathrm{~g} /$ day processed meat. In addition, a recent meta-analysis on colorectal adenoma, a precursor of colorectal cancer, observed a $36 \%(95 \% \mathrm{CI}=17-58 \%)$ increased risk for every $100 \mathrm{~g} /$ day red meat intake and $28 \%(95 \% \mathrm{CI}=3-$ $60 \%$ ) increased risk for every $50 \mathrm{~g} /$ day processed meat 
intake [10]. To our knowledge, no randomized controlled trial exclusively on red meat and processed meat modification with colorectal cancer incidence or mortality as endpoints has published results. The Polyp Prevention Trial, an intervention trial of low fat and high fruit and vegetable modification, observed a $30 \%$ reduction in red meat intake in the intervention group after 4 years of follow-up, but the study did not find a lower risk of colorectal adenoma recurrence [11]. In addition, the Women's Health Initiative Randomized Controlled Dietary Modification Trial, an intervention trial of low fat, high fruit, vegetables, and grains where a $9 \%$ reduction in red meat intake was reported, also reported no association with colorectal cancer risk after 8.1 years of follow-up [12]. However, it is possible that changes in red meat intake were too small or the followup was too short to detect a difference in risk of colorectal neoplasia in these studies, because it is not known with certainty the latency period or level of reduction in red meat intake that may bring about a reduction in colorectal neoplasia risk. A secondary analysis of the Polyp Prevention Trial did, however, find a reduction in colorectal adenoma recurrence among persons with a high compliance to the overall intervention [13].

The World Cancer Research Fund and American Institute of Cancer Research (WCRF/AICR) concluded in their 2007 report "Food, Nutrition, Physical Activity, and the Prevention of Cancer: a Global Perspective" that a high intake of red and processed meat convincingly increases the risk of colorectal cancer [14]. This conclusion was received with some criticisms $[15,16]$. Two meta-analyses of prospective studies concluded that the available epidemiologic data were not sufficient to support an independent and unequivocal positive association $[17,18]$. The controversy concerned the possible presence of confounding from other lifestyle and dietary factors in the weak magnitude of association and the inconsistency of effect by cancer subsite and gender.

Several potential mechanisms have been hypothesized to explain the link between red meat and processed meat and colorectal cancer. These include the formation of carcinogenic heterocyclic amines (HCAs) and polycyclic aromatic hydrocarbons (PAHs) in meat during high temperature cooking [19]. However, such mutagens can be formed in both white meat and red meat, but the positive association with colorectal cancer is related to red meat, not white meat [20]. A second hypothesis concerns the catalytic actions of heme iron in red meat in the induction of lipid peroxidation [21] and the endogenous formation of carcinogenic N-nitroso compounds (NOCs) in the gastrointestinal tract [22]. Nitrites or nitrates, added to meat for preservation, also could increase exposure to NOCs [23]. HCAs, PAHs, heme iron, nitrate, and nitrite may all play a role in colorectal carcinogenesis, but the exact mechanistic pathway is unclear $[14,24 \bullet \cdot]$.
We reviewed the case-control and prospective cohort studies on humans that were published in 2011 and 2012 to examine how the new findings contribute to the current bodies of evidence and we further drew implications.

\section{Red Meat, Processed Meat, Meat-Related Mutagen Intakes, and Colorectal Cancer Risk}

\section{Total Red Meat and Processed Meat}

Three large-scale prospective cohort studies - the Japan Public Health Center-based Prospective Study (JPHC) [25••], the National Institute of Health-American Association of Retired Persons (NIH-AARP) Diet and Health Study $[26 \bullet \bullet$, and the Multiethnic Cohort Study (MEC) [27••]published results on total red meat and processed meat and colorectal cancer risk in the past 2 years.

Published in the Asia Pacific Journal of Clinical Nutrition in 2011, the JPHC study with 1,145 colorectal cancer cases (788 colon and 357 rectal cancer cases) from 758,116 person-years reported a statistically significant positive association between red meat and processed meat intake (beef and pork dishes, ham, sausage, bacon, luncheon meat, and chicken liver) and colon cancer risk in women but not in men (multivariate adjusted relative risk (RR) for $93 \mathrm{~g} /$ day vs. $14 \mathrm{~g} /$ day $=1.48,95 \% \mathrm{CI}=1.01-2.17, P$ for trend $=0.03$; RR for $102 \mathrm{~g} /$ day vs. $15 \mathrm{~g} /$ day $=1.27,95 \% \mathrm{CI}=0.93-1.74$, $P$ for trend $=0.15$ respectively) $[25 \bullet \cdot$. No association was observed for rectal cancer. Further adjustment for saturated fat intake attenuated the association in women $(\mathrm{RR}=1.38$, $95 \% \mathrm{CI}=0.84-2.27, P$ for trend $=0.18)$. When analyzed by colon cancer subsite (379 proximal and 367 distal colon cancer cases), positive but statistically nonsignificant associations were observed for proximal colon cancer among women (RR for Q5 vs. Q1 $=1.57,95 \% \mathrm{CI}=0.95-1.58, P$ for trend $=0.08)$ and for distal colon cancer among men $(\mathrm{RR}$ for $\mathrm{Q} 5$ vs. $\mathrm{Q} 1=1.42,95 \% \mathrm{CI}=0.92-2.19, P$ for trend $=0.12$ ). Currently, evidence by proximal and distal colon cancers is relatively limited, but the observed associations are stronger for distal colon cancer [28-31]. Traditionally, meat consumption was low in Japan, but diet is becoming increasingly westernised. In this Japanese study, the highest quartile of red meat $(102 \mathrm{~g} /$ day in men and $93 \mathrm{~g}$ /day in women) is now comparable to an intermediate level of intake in western countries. For example, in the European Prospective Investigation into Cancer and Nutrition (EPIC) study, the highest intake category of red meat consumption was more than $160 \mathrm{~g} /$ day for men and women combined [29]; in the Nurses' Health Study (NHS, women only study) and the Health Professionals Follow-up Study (HPFS, men only study), mean consumption of the highest quartiles were 3.1 servings/day and 2.36 servings/day, respectively [32•]. 
Further supporting evidence on the influence of total red meat and processed meat intake in the risk of colorectal cancer came from the NIH-AARP Diet and Health Study. Previously, a $24 \%$ increased risk of colorectal cancer for the highest versus lowest recent red meat intake (assessed at study baseline) was observed in this elderly cohort [33]. The new article of the same study (292,797 participants, mean age 62.8 years), published in the American Journal of Clinical Nutrition in 2011, presented novel results on life course exposures [26••]. The goal was to explore the hypothesis that diet during adolescence (12-13 years old) and middle-age (approximately 10 years before study baseline) may be associated with colorectal cancer, in which the multistage adenoma-carcinoma sequence has a natural history of several decades [34]. Adolescence red meat and processed meat intake was not associated with colon or rectal cancer risk in older adulthood, whereas middle-age intake was associated with the risk of colon cancer (2,794 cases). The multivariate adjusted RR for 1.49 times/day vs. 0.18 times/day was 1.31 ( $95 \% \mathrm{CI}=1.12$ $1.53, P$ for trend $<0.01)$. No association was observed for rectal cancer (979 cases). When high adolescence and high recent adulthood intake was compared with low consumption at both lifetime periods, positive associations with colon and rectal cancers were observed $(\mathrm{RR}=1.38,95 \% \mathrm{CI}=1.16-1.64$; $\mathrm{RR}=1.39,95 \% \mathrm{CI}=1.04-1.85$ respectively), whereas slightly lower RRs were observed for persons who modified their meat consumption between adolescence and adulthood. This suggested that dietary pattern over the life course may play a role in the development of colorectal cancer. Confirmation in future studies is needed. However, there is a lack of prospective cohorts with assessment of diet during childhood and adolescence. On the other hand, recall of a distant past diet is prone to bias [35].

The MEC study published in the International Journal of Cancer in 2012 reported different results [27••]. Higher red meat and processed meat intake was associated with an increased risk of colorectal cancer in the model adjusted for age, sex, and ethnicity. However, the association disappeared after further adjustments for dietary, lifestyle, and other risk factors (multivariate adjusted RR for $47.99 \mathrm{~g} /$ $1,000 \mathrm{kcal} /$ day vs. $7.41 \mathrm{~g} / 1,000 \mathrm{kcal} /$ day $=1.02,95 \%$ $\mathrm{CI}=0.91-1.16, P$ for trends $=0.757)$. In this U.S. study, 3,404 colorectal cancer cases from 165,717 men and women of different ethnic background were accrued during a follow-up of 13.6 years. Total red meat and processed meat intake was collected using a quantitative food frequency questionnaire (QFFQ). The null findings (including those of red meat or processed meat) were previously reported in another publication of the same study (nested case-control design, 1,009 cases, 1,522 controls) [36] and were included in our meta-analyses [9].

In addition to the cohort studies, a Jordanian hospitalbased case-control study, published in the Asian Pacific
Journal of Cancer Prevention in 2011, also has observed a statistically significant positive association between red meat intake and colorectal cancer risk [37]. The odds ratio (OR) for the highest compared with the lowest consumption was 2.66 (95\% CI=1.83-3.88). The 220 cases and 220 controls in this study were matched for age and sex. Dietary data in the past 12 months were collected during an interview that was performed 10-14 days after cancer diagnosis. Red meat intake was described to be high in Jordan [37].

\section{Fresh Red Meat}

In the JPHC study, beef and pork intakes were reported to increase colon cancer risk in women (RRs for Q5 vs. Q1= $1.62,95 \% \mathrm{CI}=1.12-2.34, P$ for trend $=0.04$ and $1.42,95 \%$ $\mathrm{CI}=0.99-2.04, P$ for trend $=0.05$ respectively) $[25 \bullet \bullet]$. The same elevated risk was neither shown with rectal cancer, nor in men for both colorectal cancer subsites. In Japan, beef and pork is a major source of fresh red meat intake. In the MEC study, similar to total red meat and processed meat, the positive association of red meat excluding processed meat intake with colorectal cancer risk disappeared after multivariate adjustments [27••]. The RR for $34.86 \mathrm{~g} / 1,000 \mathrm{kcal} /$ day vs. $4.59 \mathrm{~g} / 1,000 \mathrm{kcal} /$ day was $0.98(95 \% \mathrm{CI}=0.87-1.1, P$ for trends $=0.584)$. The new publication on the NIH-AARP study did not report results on adolescence or middle-age fresh red meat intake [26•*]. An earlier publication of this study on recent diet observed a RR of $1.13(95 \% \mathrm{CI}=0.98-1.3, P$ for trend $=0.002$ ) for the comparison of the highest with the lowest non-processed red meat intake [20]. In addition, two case-control studies from Spain and Japan published results in $2012[38,39]$. The Spanish hospital-based case-control study observed an increased risk of colorectal cancer with red meat intake (OR for T3 vs. T1 $=1.79,95 \% \mathrm{CI}=1.02-3.12, P$ for trend $=0.03$ ) [38]. This analysis of 245 cases and 490 controls was adjusted for age, sex, and energy intake only. Diet six months before cancer diagnosis was assessed using a 142-item FFQ and mean red meat intakes were $21.4 \mathrm{~g}$ /day for the cases and $15.2 \mathrm{~g} /$ day for the controls. In the Japanese multiinstitutional case-control study of 1,511 cases and 2,098 controls, a positive association between beef or pork intake and colorectal cancer risk was observed in men (age, sex, and location adjusted OR for $\geq 3$ times/week vs. $\leq 2$ times/week $=$ $1.26,95 \% \mathrm{CI}=1.09-1.47)$, but not in women $(\mathrm{OR}=0.94$, $95 \% \mathrm{CI}=0.79-1.12)[39]$.

\section{Processed Meat}

Processed meat intake was not associated with colorectal cancer risk in the JPHC study [25 • ]. Processed meat intake remained low (average $10 \mathrm{~g} /$ day), despite red meat consumption being relatively high in this Japanese cohort. Low intake of processed meat may explain the lack of an association. On the 
contrary, the NIH-AARP study reported that processed meat intake during middle-age was associated with the risk of colon and rectal cancers in older adulthood (multivariate adjusted RRs for 1.02 times/day vs. 0.05 times/day $=1.24$, $95 \% \mathrm{CI}=1.06-1.45, P$ for trend $<0.01 ; \mathrm{RR}=1.3,95 \% \mathrm{CI}=$ $0.99-1.7, P$ for trend $=0.16$ respectively) [26••]. In addition, high consumption during adolescence and recent adulthood compared with low consumption at both lifetime periods was positively associated with colon cancer $(\mathrm{RR}=1.25,95 \% \mathrm{CI}=$ 1.06-1.47), but not with rectal cancer (data not shown). Adolescence intake was not associated with colon or rectal cancers. As for the MEC study, no association was observed (multivariate adjusted RR for $17.98 \mathrm{~g} / 1,000 \mathrm{kcal} /$ day vs. $1.7 \mathrm{~g} / 1,000 \mathrm{kcal} /$ day was $1.06,95 \% \mathrm{CI}=0.94-1.19, P$ for trends $=0.26$ ) $[27 \bullet \bullet]$. The positive association is supported by a Uruguayan hospital-based, multisite, case-control study published in the British Journal of Cancer in 2012 [40]. Higher processed meat intake was found to increase colorectal cancer risk (multivariate adjusted $\mathrm{OR}$ for $\geq 28.3 \mathrm{~g}$ /day vs. $\leq 11.4 \mathrm{~g} /$ day $=2.39,95 \% \mathrm{CI}=1.76-3.24)$. The magnitude of the association was similar for colon (176 cases) and rectal (185 cases) cancers. Mean total processed meat intake ranged from 19.9 to $38.6 \mathrm{~g} /$ day. Of interest is that the risk associated with the highest versus the lowest processed meat intake in this study is stronger with colorectal cancer than with other cancer sites, such as breast and prostate, and of similar magnitude as other cancers of the aerodigestive system. The NIH-AARP study also reported on multiple cancers [33]. Positive associations with processed meat intake were observed for pancreatic and lung cancers in this study.

\section{Cooking Methods and Heterocyclic Amines}

Previous epidemiological evidence on cooking methods, total and specific HCAs, and colorectal cancer risk are variable [20, 41]. In 2011, a population-based case-control study (the Western Australian Bowel Health Study [WABHS] published in the European Journal of Clinical Nutrition) reported no association between total red meat consumption (on average $325 \mathrm{~g} /$ day), cooking methods (pan fried, BBQ, baked, stewed, and microwave), and level of doneness and colorectal cancer risk [42]. There were 713 controls matched by age and sex to 567 incident cases in this study, with relatively low response rates $(46.5 \%$ and $59.5 \%$ respectively). Habitual diet 10 years ago was assessed with a self-administered, 74-item, semiquantitative FFQ. In addition, the MEC study reported that higher intake of total meat cooked dark brown or well done, total, and specific HCAs also was not associated with colorectal cancer risk [27••]. HCA intakes (specifically PhlP, DiMelQx, and MelQx) were derived from the CHARRED database based on data on type of meat, cooking method, frequency of consumption, and doneness level.
N-nitroso-Compounds and Nitrites

Few observational studies on humans have published results on NOCs, nitrate, and nitrite intake in relation to colorectal cancer risk. Evidence from experimental studies support a potential link, in which a high level of fecal nitrosocompounds was found to promote carcinogenesis in rats fed with cured meat [24••]. The two prospective studies on humans published before 2011 observed positive associations with dietary nitrate from processed meat [20] and Nnitrosodimethylamine (NDMA) [43]. In 2011, the EPICNorfolk, UK study published results in the American Journal of Clinical Nutrition [44-]. A total of 23,363 participants were followed up prospectively for an average of 11.4 years. Dietary NDMA and nitrite consumption was estimated by matching FFQ food items with a food database of potential carcinogens. It was shown that dietary NDMA was statistically significantly associated with increased rectal cancer risk (multivariate adjusted RR for each 1standard deviation increase $=1.46,95 \% \mathrm{CI}=1.16-1.84$, $P=0.001,137$ cases). No association was observed for colon cancer risk (276 cases). Analyses of endogenous N-nitroso compound exposure index (ENOC) and dietary nitrite also found no relation to colon or rectal cancer. Of note, dietary NDMA was not solely contributed by processed meat intake; beer also was a major source in this population.

\section{Heme Iron}

A meta-analysis of five prospective cohort studies $(4,734$ colon cancer cases, 566,607 participants) published in Cancer Prevention Research in 2011 observed an $18 \%$ (95\% $\mathrm{CI}=6-32 \%$ ) increased risk for colon cancer comparing the highest with the lowest heme iron intake [45•]. There was no evidence of heterogeneity between studies ( $P$ for heterogeneity $=0.18)$. However, two recent publications from three prospective cohort studies (NHS and HPFS, and JPHC) showed no association between heme iron intake and colorectal cancer risk (for the highest vs. lowest comparison, multivariate adjusted $\mathrm{RR}=1.21,95 \% \mathrm{CI}=0.96-1.52, P$ for trend $=0.1$ in NHS; RR $=0.98,95 \% \mathrm{CI}=0.77-1.26, P$ for trend $=0.8$ in HPFS; RR $=1.06,95 \% \mathrm{CI}=0.79-1.42, P$ for trend $=0.6$ in men and $\mathrm{RR}=0.88,95 \% \mathrm{CI}=0.61-1.29, P$ for trend $=0.4$ in women in JPHC) $[46 \bullet, 47 \cdot]$. Heme iron intake comparisons (mg/day) were 1.6 vs. 0.7 in the NHS, 1.8 vs. 0.8 in HPFS, and 0.77 vs. 0.24 and 0.67 vs. 0.23 in JPHC. Large numbers of colorectal cancer cases were accrued in these three studies: 1,079 cases among 69,345 women in NHS, 1,035 cases among 45,716 men in HPFS, and 786 cases among 39,721 men and 498 cases among 45,376 women in JPHC. Animal foods are the sources of dietary heme iron. In the Japanese study, $49 \%$ of heme iron was derived from fish and shellfish [46•], whereas in the U.S. 
studies, the main source of heme iron was red meat and chicken (approximately 60-70 \%) [47•].

\section{Conclusions}

During the past decades, a large number of epidemiological studies on humans have examined the association between red meat and/or processed meat intake and the risk of incident colorectal cancer in different populations. This includes studies with different designs [24••]. Evidence from randomized controlled trials is limited because of implementation difficulties and ethical concerns. Findings are mostly accumulated from observational investigations, of which cohort studies are less prone to bias than casecontrol studies [48]. Well-conducted, large-scale prospective cohorts with a long follow-up, for instance, NHS, HPFS, NIH-AARP Diet and Health Study, MEC, Iowa Women's Health Study, Cancer Prevention Study II, The Netherlands Cohort Study, Swedish Mammography Cohort, EPIC, Melbourne Collaborative Cohort Study, JPHC, and Shanghai Women's Health Study, have contributed results. Previous dose-response and highest versus lowest metaanalyses of published data showed a moderate increased risk for colorectal cancer with total red meat and processed meat, fresh red meat, and processed meat [9]. There was little heterogeneity between studies on fresh red meat and processed meat, but moderate heterogeneity between studies on total red meat and processed meat was observed. The magnitude of associations with fresh red meat and processed meat are comparable in the meta-analysis. Results from studies published in 2011 and 2012 are inconsistent, but generally point toward an increased risk. Studies on meat constituents and related carcinogens hypothesized to have putative roles in colorectal cancer development, although not entirely consistent, have suggested a positive association with heme iron intake, whereas results for meat-related mutagens are more variable.

The inconsistency could be explained partly by the limitations of accurately measuring diet and other compounds. Regression dilution bias due to the lack of updated exposure data in prospective studies and measurement error in the assessment of meat intake are particularly problematic and could attenuate the risk estimate toward the null as shown in previous studies $[29,32 \cdot, 49,50]$. Small numbers of cases, in particular in some subgroup analyses, and a small range of meat intake may hinder an individual study's ability to detect modest associations [27••, 51]. Failure to adjust for relevant covariates and residual confounding are critical issues when determining diet-cancer associations; nevertheless, recent studies mostly employed multivariate models and accounted for known confounders. Genetic polymorphisms also may modify the association of red meat and processed meat with colorectal cancer risk [52]. Future studies should try to collect, with repeated measurements, accurate estimates of meat intake and related cooking information in a large range of populations. The development of biomarkers for meat consumption is being researched [53].

Current American Cancer Society guidelines for cancer prevention are to limit processed meat and red meat consumption and to prepare meat by baking, broiling, or poaching rather than by frying or charbroiling (http://www.cancer.org/ acs/groups/cid/documents/webcontent/002577-pdf.pdf) [54]. The U.K. Department of Health recommends high red and processed meat consumers $(\geq 90 \mathrm{~g} /$ day) to reduce intake to $70 \mathrm{~g} /$ day (http://www.nhs.uk/Livewell/Goodfood/ Pages/meat.aspx\#red) [55]. The World Cancer Research Fund recommends the limitation of red meat intake $(<500 \mathrm{~g} /$ week) and the avoidance of processed meat consumption (http://www.wcrf-uk.org/cancer_prevention/recommendations/ meat_and_cancer.php) [56]. Adherence to dietary guidelines for red and processed meat consumption reduced colorectal cancer risk $[57,58]$. In addition, a western dietary pattern that is characterized by a higher red and processed meat consumption was associated with an increased colon cancer risk [59]. The recent evidence is overall supportive of an increased risk in colorectal cancer with higher consumption of red meat and processed meat. It is prudent to adhere to the recommended dietary guidelines.

Disclosure No potential conflicts of interest relevant to this article were reported.

\section{References}

Papers of particular interest, published recently, has been highlighted as:

- Of importance

•- Of major importance

1. Ferlay J, Shin HR, Bray F, et al. Estimates of worldwide burden of cancer in 2008: GLOBOCAN 2008. Int J Cancer. 2010;127:2893-917.

2. Joshu CE, Parmigiani G, Colditz GA, et al. Opportunities for the primary prevention of colorectal cancer in the United States. Cancer Prev Res (Phila). 2012;5:138-45.

3. Bejar LM, Gili M, Infantes B, et al. Incidence of colorectal cancer and influence of dietary habits in fifteen European countries from 1971 to 2002. Gac Sanit. 2012;26:69-73.

4. Bingham S, Riboli E. Diet and cancer-the European Prospective Investigation into Cancer and Nutrition. Nat Rev Cancer. 2004;4:206-15.

5. McKeown-Eyssen GE, Bright-See E. Dietary factors in colon cancer: international relationships. Nutr Cancer. 1984;6:160-70.

6. Larsson SC, Wolk A. Meat consumption and risk of colorectal cancer: a meta-analysis of prospective studies. Int $\mathrm{J}$ Cancer. 2006;119:2657-64. 
7. Norat T, Lukanova A, Ferrari $\mathrm{P}$, et al. Meat consumption and colorectal cancer risk: dose-response meta-analysis of epidemiological studies. Int J Cancer. 2002;98:241-56.

8. Sandhu MS, White IR, McPherson K. Systematic review of the prospective cohort studies on meat consumption and colorectal cancer risk: a meta-analytical approach. Cancer Epidemiol Biomarkers Prev. 2001;10:439-46.

9. Chan DS, Lau R, Aune D, et al. Red and processed meat and colorectal cancer incidence: meta-analysis of prospective studies. PLoS One. 2011;6:e20456.

10. $\mathrm{Xu} \mathrm{X,} \mathrm{Yu} \mathrm{E,} \mathrm{Gao} \mathrm{X,} \mathrm{et} \mathrm{al.:} \mathrm{Red} \mathrm{and} \mathrm{processed} \mathrm{meat} \mathrm{intake} \mathrm{and} \mathrm{risk}$ of colorectal adenomas: A meta-analysis of observational studies. Int J Cancer 2012,

11. Mathew A, Sinha R, Burt R, et al. Meat intake and the recurrence of colorectal adenomas. Eur J Cancer Prev. 2004;13:159-64.

12. Beresford SA, Johnson KC, Ritenbaugh C, et al. Low-fat dietary pattern and risk of colorectal cancer: the Women's Health Initiative Randomized Controlled Dietary Modification Trial. JAMA. 2006;295:643-54.

13. Sansbury LB, Wanke K, Albert PS, et al. The effect of strict adherence to a high-fiber, high-fruit and -vegetable, and low-fat eating pattern on adenoma recurrence. Am J Epidemiol. 2009;170:576-84.

14. World Cancer Research Fund/American Institute of Cancer Research. Food, Nutrition, Physical Activity, and the Prevention of Cancer: a Global Perspective. Washington: AICR; 2007.

15. Truswell AS. Problems with red meat in the WCRF2. Am J Clin Nutr. 2009;89:1274-5.

16. Boyle P, Boffetta P, Autier P. Diet, nutrition and cancer: public, media and scientific confusion. Ann Oncol. 2008;19:1665-7.

17. Alexander DD, Miller AJ, Cushing CA, et al. Processed meat and colorectal cancer: a quantitative review of prospective epidemiologic studies. Eur J Cancer Prev. 2010;19:328-41.

18. Alexander DD, Weed DL, Cushing CA, et al. Meta-analysis of prospective studies of red meat consumption and colorectal cancer. Eur J Cancer Prev. 2011;20:293-307.

19. Sugimura T, Wakabayashi K, Nakagama H, et al. Heterocyclic amines: Mutagens/carcinogens produced during cooking of meat and fish. Cancer Sci. 2004;95:290-9.

20. Cross AJ, Ferrucci LM, Risch A, et al. A large prospective study of meat consumption and colorectal cancer risk: an investigation of potential mechanisms underlying this association. Cancer Res. 2010;70:2406-14.

21. Nelson RL. Iron and colorectal cancer risk: human studies. Nutr Rev. 2001;59:140-8

22. Bingham SA, Pignatelli B, Pollock JR, et al. Does increased endogenous formation of $\mathrm{N}$-nitroso compounds in the human colon explain the association between red meat and colon cancer? Carcinogenesis. 1996;17:515-23.

23. Santarelli RL, Pierre F, Corpet DE. Processed meat and colorectal cancer: a review of epidemiologic and experimental evidence. Nutr Cancer. 2008;60:131-44.

24. - Corpet DE. Red meat and colon cancer: should we become vegetarians, or can we make meat safer? Meat Sci. 2011;89:310-6. This paper is a concise review on red meat and processed meat intake and its postulated underlying mechanisms in colorectal cancer development, with detailed evidence from human and animal studies.

25. • Takachi R, Tsubono $\mathrm{Y}, \mathrm{Baba} \mathrm{K}$, et al. Red meat intake may increase the risk of colon cancer in Japanese, a population with relatively low red meat consumption. Asia Pac J Clin Nutr. 2011;20:603-12. This paper is a large Japanese cohort in which, by Western standards, red meat consumption was considered moderate. Positive association between red meat intake and colon cancer risk was observed in women.

26. •- Ruder EH, Thiebaut AC, Thompson FE, et al. Adolescent and mid-life diet: risk of colorectal cancer in the NIH-AARP Diet and Health Study. Am J Clin Nutr. 2011;94:1607-19. Novel results on life course exposures were provided in this large elderly cohort. High red and processed meat intake during middle-age elevated the risk of colorectal cancer.

27. • Ollberding NJ, Wilkens LR, Henderson BE, et al. Meat consumption, heterocyclic amines and colorectal cancer risk: the Multiethnic Cohort Study. Int J Cancer. 2012;131:E1125-33. This large multi-ethnic cohort found no association between red meat and processed meat intake and colorectal cancer risk in the analyses adjusted for multiple confounders. Total or specific HCAs were also not related to colorectal cancer risk.

28. Sato Y, Nakaya N, Kuriyama S, et al. Meat consumption and risk of colorectal cancer in Japan: the Miyagi Cohort Study. Eur J Cancer Prev. 2006;15:211-8.

29. Norat T, Bingham S, Ferrari P, et al. Meat, fish, and colorectal cancer risk: the European Prospective Investigation into cancer and nutrition. J Natl Cancer Inst. 2005;97:906-16.

30. Giovannucci E, Rimm EB, Stampfer MJ, et al. Intake of fat, meat, and fiber in relation to risk of colon cancer in men. Cancer Res. 1994;54:2390-7.

31. Larsson SC, Rafter J, Holmberg L, et al. Red meat consumption and risk of cancers of the proximal colon, distal colon and rectum: the Swedish Mammography Cohort. Int J Cancer. 2005;113:829-34.

32. - Pan A, Sun Q, Bernstein AM, et al. Red meat consumption and mortality: results from 2 prospective cohort studies. Arch Intern Med. 2012;172:555-63. This paper on red meat intake and morality from two large American cohorts demonstrated that measurement error weakens the association.

33. Cross AJ, Leitzmann MF, Gail MH, et al. A prospective study of red and processed meat intake in relation to cancer risk. PLoS Med. 2007;4:e325.

34. Fearon ER, Vogelstein B. A genetic model for colorectal tumorigenesis. Cell. 1990;61:759-67.

35. Dwyer JT, Coleman KA. Insights into dietary recall from a longitudinal study: accuracy over four decades. Am J Clin Nutr. 1997;65:1153S-8.

36. Nothlings U, Yamamoto JF, Wilkens LR, et al. Meat and heterocyclic amine intake, smoking, NAT1 and NAT2 polymorphisms, and colorectal cancer risk in the multiethnic cohort study. Cancer Epidemiol Biomarkers Prev. 2009;18:2098-106.

37. Arafa MA, Waly MI, Jriesat S, et al. Dietary and lifestyle characteristics of colorectal cancer in Jordan: a case-control study. Asian Pac J Cancer Prev. 2011;12:1931-6.

38. Banque M, Raido B, Masuet C, et al. Food groups and nutrient intake and risk of colorectal cancer: a hospital-based case-control study in Spain. Nutr Cancer. 2012;64:386-92.

39. Ishimaru S, Mimori $\mathrm{K}$, Yamamoto $\mathrm{K}$, et al. Increased risk for $\mathrm{CRC}$ in diabetic patients with the nonrisk allele of SNPs at 8q24. Ann Surg Oncol. 2012;19:2853-8.

40. De Stefani E, Boffetta P, Ronco AL, et al. Processed meat consumption and risk of cancer: a multisite case-control study in Uruguay. Br J Cancer. 2012;107:1584-8.

41. Cross AJ, Sinha R. Meat-related mutagens/carcinogens in the etiology of colorectal cancer. Environ Mol Mutagen. 2004;44:44-55.

42. Tabatabaei SM, Fritschi L, Knuiman MW, et al. Meat consumption and cooking practices and the risk of colorectal cancer. Eur J Clin Nutr. 2011;65:668-75.

43. Knekt P, Jarvinen R, Dich J, et al. Risk of colorectal and other gastrointestinal cancers after exposure to nitrate, nitrite and N-nitroso compounds: a follow-up study. Int J Cancer. 1999;80:852-6.

44. • Loh YH, Jakszyn P, Luben RN, et al. N-Nitroso compounds and cancer incidence: the European Prospective Investigation into Cancer and Nutrition (EPIC)-Norfolk Study. Am J Clin Nutr. 2011;93:1053-61. This paper is a large multi-center European study that explored the link between heme iron intake and cancer risk. Positive association with dietary NDMA was observed in separate analyses on rectal cancer, but not colon cancer. 
45. - Bastide NM, Pierre FH, Corpet DE. Heme iron from meat and risk of colorectal cancer: a meta-analysis and a review of the mechanisms involved. Cancer Prev Res (Phila). 2011;4:177-84. This paper is a meta-analysis of prospective cohort studies on heme iron intake and colon cancer risk, with detailed explanation on the mechanisms involved.

46. - Hara A, Sasazuki S, Inoue M, et al. Zinc and heme iron intakes and risk of colorectal cancer: a population-based prospective cohort study in Japan. Am J Clin Nutr. 2012;96:864-73. This large Japanese cohort found no association between heme iron intake and colorectal cancer risk.

47. - Zhang X, Giovannucci EL, Smith-Warner SA, et al. A prospective study of intakes of zinc and heme iron and colorectal cancer risk in men and women. Cancer Causes Control. 2011;22:162737. This paper presented results from two large American cohorts that observed no association between heme iron intake and colorectal cancer risk.

48. Colditz GA. Overview of the epidemiology methods and applications: strengths and limitations of observational study designs. Crit Rev Food Sci Nutr. 2010;50 Suppl 1:10-2.

49. Larsson SC, Hakanson N, Permert J, et al. Meat, fish, poultry and egg consumption in relation to risk of pancreatic cancer: a prospective study. Int J Cancer. 2006;118:2866-70.

50. Pan A, Sun Q, Bernstein AM, et al. Red meat consumption and risk of type 2 diabetes: 3 cohorts of US adults and an updated meta-analysis. Am J Clin Nutr. 2011;94:1088-96.
51. Spencer EA, Key TJ, Appleby PN, et al. Meat, poultry and fish and risk of colorectal cancer: pooled analysis of data from the UK dietary cohort consortium. Cancer Causes Control. 2010;21:1417-25.

52. Shin A, Kim J. Effect modification of meat intake by genetic polymorphisms on colorectal neoplasia susceptibility. Asian Pac J Cancer Prev. 2010;11:281-7.

53. Cross AJ, Major JM, Sinha R. Urinary biomarkers of meat consumption. Cancer Epidemiol Biomarkers Prev. 2011;20:1107-11.

54. American Cancer Society: American Cancer Society guidelines on nutrition and physical activity for cancer prevention. Available at http:// www.cancer.org/acs/groups/cid/documents/webcontent/002577-pdf.pdf

55. National Health Service Choices: Meat. Available at http:// www.nhsuk/Livewell/Goodfood/Pages/meat.aspx\#red

56. World Cancer Research Fund: Red and processed meats and cancer prevention. Available at http://www.wcrf-uk.org/cancer_prevention/ recommendations/meat_and_cancer.php

57. Kirkegaard H, Johnsen NF, Christensen J, et al. Association of adherence to lifestyle recommendations and risk of colorectal cancer: a prospective Danish cohort study. BMJ. 2010;341:c5504.

58. Romaguera D, Vergnaud AC, Peeters $\mathrm{PH}$, et al. Is concordance with World Cancer Research Fund/American Institute for Cancer Research guidelines for cancer prevention related to subsequent risk of cancer? Results from the EPIC study. Am J Clin Nutr. 2012;96:150-63.

59. Magalhaes B, Peleteiro B, Lunet N. Dietary patterns and colorectal cancer: systematic review and meta-analysis. Eur J Cancer Prev. 2012;21:15-23. 